\title{
VALORES DE COMPRA HEDÔNICO E UTILITÁRIO: OS ANTECEDENTES E AS RELAÇÕES COM OS RESULTADOS DO VAREJO
}

\author{
João Marques Teixeira \\ joaomarquest@estadao.com.br \\ Centro Universitário Nove de Julho - São Paulo, SP/Brasil \\ José Mauro da Costa Hernandez \\ jmhernandez@ @ei.edu.br \\ Centro Universitário Nove de Julho - São Paulo, SP/Brasil
}

\begin{abstract}
Recebido em 25/05/2009
Aprovado em 20/12/2011

Disponibilizado em 01/04/2012

Avaliado pelo sistema double blind review

Revista Eletrônica de Administração

Editor: Luís Felipe Nascimento

ISSN 1413-2311 (versão on-line)

Editada pela Escola de Administração da Universidade Federal do Rio Grande do Sul.

Periodicidade: Quadrimestral
\end{abstract}

Sistema requerido: Adobe Acrobat Reader.

\section{RESUMO}

Motivado pelo crescente debate sobre a influência que o ambiente de uma loja pode exercer nos consumidores e pela enorme quantidade de recursos que os varejistas devotam para criar clientes satisfeitos e leais, este artigo propõe um novo modelo em que a relação entre as variáveis ambientais da loja (ambiente físico, organização, sortimento e atendimento dos vendedores) e as variáveis de resultado do varejo (satisfação com a compra, intenção de recompra e intenção de boca a boca) é intermediada pelos valores de compra hedônico e utilitário. Para testar o modelo teórico, foram realizadas 399 entrevistas com pessoas que adquiriram eletrodomésticos em lojas de uma rede de varejo na cidade de São Paulo, Brasil. Os resultados indicaram que, dentre as variáveis ambientais da loja, apenas o atendimento dos vendedores influenciou positivamente os valores de compra hedônico e utilitário. Por sua vez, o valor de compra hedônico influenciou positivamente todas as variáveis de resultado do varejo enquanto que o valor de compra utilitário não influenciou nenhuma da variáveis de resultado do varejo. Os resultados sugerem que, neste caso, o valor hedônico foi muito mais importante que o valor utilitário para a satisfação com a compra, a intenção de voltar à loja e a intenção de engajar-se em boca a boca positivo. Os resultados também sugerem a grande importância do atendimento dos vendedores em situações de varejo em que o ambiente da loja não se revela particularmente atraente ou diferenciado.

Palavras-chave: Valor de compra hedônico, valor de compra utilitário, ambiente físico de loja, satisfação, boca a boca, intenção de recompra. 


\title{
HEDONIC AND UTILITARIAN SHOPPING VALUES: ANTECEDENTS AND RELATIONSHIP WITH RETAIL OUTCOMES
}

\begin{abstract}
Induced by the recent debate regarding the influence that the store environment can have on consumers and by the massive amount of resources that retailers have spent in order to create satisfied consumers, this study proposes a new model in which the relationship between store environment variables (physical environment, organization, assortment, and salesman service) and retail outcomes variables (satisfaction, repurchase intention, and word-of-mouth) is mediated by hedonic and utilitarian shopping values. To test the theoretical model, data were collected from the 399 respondents who purchased appliances at a store located in the city of São Paulo, Brazil. The results indicated that among the environmental variables of the store, only the assistance of salesman has positively influenced the values of hedonic and utilitarian shopping. Hedonic shopping value influenced positively all the retail outcomes variables while utilitarian shopping value did not influence any of them. The results suggest that in this particular case, hedonic shopping value was much more important than utilitarian shopping value to determine purchase satisfaction, repurchase intention, and intention of word-of-mouth. The results also suggest the greater importance of salesman service in situations that the store environment is not especially attractive or different.
\end{abstract}

Keywords: Hedonic shopping value, utilitarian shopping value, store physical environment, satisfaction, word-of-mouth, repurchase intention.

\section{INTRODUÇÃO}

O estudo da dimensão hedônica do consumo iniciou-se com as pesquisas de Hirschman e Holbrook (HIRSCHMAN; HOLBROOK, 1982; HOLBROOK; HIRSCHMAN, 1982). Segundo esta linha de pesquisa, o consumo hedônico suscita imagens multisensoriais, fantasias e emoções e se diferencia da visão tradicional, baseada unicamente nas atitudes e julgamentos dos consumidores em relação à utilidade "econômica" dos produtos. Hirschman; Holbrook (1982) sugeriram que, assim como os produtos têm os seus valores hedônico e utilitário intrínsecos, teoricamente os consumidores também poderiam desenvolver diferentes níveis de valores de compra hedônico e utilitário a partir de uma experiência de compra.

De modo geral, o valor hedônico de produtos é relevante para os domínios afetivos, experimentais e simbólicos do consumo porque suscita prazer e excitação enquanto que o valor utilitário de produtos é primariamente orientado para objetivos (HIRSCHMAN; HOLBROOK, 1982; HOLBROOK; HIRSCHMAN, 1982; BATRA; AHTOLA, 1991; MANO; OLIVER, 1993). Hirschman; Holbrook (1982) chegaram mesmo a sugerir que os produtos hedônicos podem desempenhar um papel importante na vida dos consumidores ao fornecer-lhes prazer e escape para as suas emoções e fantasias. Por outro lado, tendo em vista que a compra de produtos utilitários se caracteriza pela racionalidade, os consumidores 
Valores de compra hedônico e utilitário: os antecedentes e as relações com os resultados do varejo

envolvidos neste tipo de compra poderiam desenvolver atitudes neutras ou até mesmo negativas devido à racionalidade do processo.

Diversos estudos posteriores demonstraram que os consumidores avaliam e escolhem os produtos em função de seu valor hedônico e utilitário (BATRA; AHTOLA, 1991; DHAR; WERTENBROCH, 2000). Estas constatações estimularam a criação de uma escala de valor de compra (BABIN; DARDEN; GRIFFIN, 1994) baseada nas diferentes atitudes dos consumidores em relação a uma compra. O desenvolvimento desta escala acabou impulsionando outros estudos sobre os antecedentes dos valores de compra hedônico e utilitário (BABIN et al., 1994; BABIN; DARDEN, 1996; GRIFFIN; BABIN; MODIANOS, 2000; BABIN; BABIN, 2001; BABIN et al., 2005).

Entretando, estudos anteriores sobre os valores de compra não levaram em consideração a influência que o ambiente físico de uma loja pode exercer sobre os valores de compra hedônico e utilitário. Belk (1975) já havia sugerido que o ambiente físico de uma loja é tão importante quanto as características individuais dos consumidores e um dos importantes influenciadores do seu comportamento. Neste sentido, Bloch; Ridgway; Dawson (1994) mostraram que o ambiente físico de uma loja, que inclui, entre outros elementos, layout, arquitetura, decoração, iluminação, música, aromas e limpeza, influencia o estado emocional dos consumidores.

Outros estudos também mostraram que a emoção positiva suscitada por um ambiente estimulante encoraja o consumidor a permanecer por mais tempo dentro da loja (HUI; BATESON, 1991). O sortimento da loja, os preços, a equipe de vendas, o fato da loja estar ou não muito cheia e os elementos periféricos dos serviços do varejista também podem contribuir para a avaliação da natureza de uma compra (JONES, 1999; COTTET; LICHTLÉ; PLICHON, 2006). Portanto, a primeira pergunta que buscamos responder neste estudo é se o ambiente físico de uma loja pode influenciar os valores de compra hedônico e utilitário derivados de uma experiência de compra.

Uma outra linha de investigação pouco explorada na literatura de marketing diz respeito às relações entre os valores de compra e as variáveis de resultado do varejo. Embora a escala de valor de compra de Babin et al. (1994) tenha impulsionado os estudos sobre os antecedentes dos valores de compra hedônico e utilitário, poucos deles tiveram como foco a avaliação do relacionamento entre os valores de compra e as variáveis de resultado do varejo. Isto é mais surpeendente quando se leva em conta a enorme quantidade de recursos que os varejistas devotam para a criação de clientes satisfeitos e leais (CRONIN; BRADY; HULT, 2000; JONES; REYNOLDS; ARNOLD, 2006). 
Estudos anteriores indicaram que os julgamentos de satisfação dependem das experiências afetivas acumuladas com o produto e das crenças e outros conhecimentos retidos e atualizados ao longo do tempo (OLIVER, 1997). Outros estudos sugeriram que o boca a boca surge a partir do forte envolvimento emocional do consumidor com o produto (DICHTER, 1966; WESTBROOK, 1987) e das percepções de valor e avaliações de eqüidade (SWAN; OLIVER, 1989). Outras pesquisas ainda indicaram que as experiências afetivas em uma experiência de compra são importantes antecedentes das motivações de aproximação tal como a intenção de recompra (DONOVAN; ROSSITER, 1982). Portanto, a segunda pergunta que buscamos responder neste estudo é se os valores de compra influenciam as variáveis de resultado do varejo.

Dizendo de outra forma, buscamos verificar se os valores de compra hedônico e utilitário intermedeiam a relação entre as variáveis ambientais da loja e as variáveis de resultado do varejo. Para atingir este objetivo, estruturamos este estudo da seguinte forma. $\mathrm{Na}$ primeira seção, discutimos o conceito de valor, definimos valor de compras hedônico e utilitário e identificamos as relações propostas entre as variáveis ambientais de uma loja e os valores de compra e entre estes e as variáveis de resultado do varejo. Na segunda seção apresentamos o método usado para testar o modelo teórico e em seguida apresentamos os resultados. Na quarta e última seção, discutimos os resultados e apresentamos as concluões, limitações do estudo e sugestões de estudos futuros.

\section{REVISÃO DA LITERATURA}

Na revisão a seguir, inicialmente discutimos o conceito de valor e os conceitos de valor hedônico e utilitário de produtos e compras. Em seguida, cada uma das seções apresenta as hipóteses do modelo teórico que será testado.

\subsection{O conceito de valor}

Valor para o cliente é o fundamento de toda atividade de marketing (HOLBROOK, 1999) e uma das razões da existência das empresas (SLATER, 1997). Ravald; Grönroos (1996) consideram que a capacidade de proporcionar valor superior para os consumidores é uma das estratégias competitivas mais promissoras para qualquer organização. Portanto, entender o que significa valor para o cliente de forma inequívoca é questão central não apenas para acadêmicos mas também para praticantes de marketing.

Entretanto, definir valor não parecer ser tarefa simples. Por exemplo, considere os seguintes pressupostos que têm complicado a definição de valor para o cliente: valor pode 
Valores de compra hedônico e utilitário: os antecedentes e as relações com os resultados do varejo

ocorrer antes, durante e após a transação (WOODALL, 2003); valor pode resultar da aquisição ou uso de um produto ou serviço (SWEENEY; SOUTAR, 2001) ou simplesmente de uma experiência de compra (BABIN et al., 1994); valor para o consumidor (o resultado de um processo avaliativo) deve ser diferenciado dos valores do consumidor (padrões, regras, critérios, normas, objetivos ou ideais que servem de base para tal julgamento avaliativo) (HOLBROOK, 1999); valor varia de contexto para contexto (uma garrafa de água no deserto não tem o mesmo valor na cidade) e é subjetivo (ZEITHAML, 1988).

As primeiras definições de valor para o cliente na literatura de marketing (ZEITHAML, 1988; MONROE, 1979) foram baseadas na visão econômica, segundo a qual o consumidor é um ser racional que tenta maximizar a sua utilidade tendo em vista as restrições de preço dos bens e da sua renda. De acordo com esta visão, portanto, o valor é derivado a partir da diferença entre a utilidade proporcionada pelos atributos de um produto e a "desutilidade" representada pelo preço pago (SÁNCHEZ-FERNÁNDEZ; INIESTA-BONILLO, 2007). Assim, Zeithaml (1988, p. 14) define valor como "a avaliação global do consumidor da utilidade de um produto baseada em percepções daquilo que é recebido e daquilo que é dado" enquanto que para Monroe (1979, p. 46), “a percepção de valor para o cliente representa uma relação entre a qualidade e os benefícios percebidos do produto e os sacrifícios percebidos ao pagar pelo produto".

Apesar do mérito da simplicidade, a abordagem econômica de valor para o cliente como uma troca entre benefícios e sacrifícios foi muito criticada por não levar em conta fatores intangíveis, intrínsecos e emocionais que formam o construto (MATWICK; MALHOTRA; RIGDON, 2001). Definições mais recentes (SHETH; NEWMAN; GROSS, 1991; WOODRUFF, 1997; HOLBROOK, 1999) buscaram inspiração em outras ciências que não a econômica para oferecer visões menos restritivas para o conceito de valor para o cliente.

Assim, Woodruff (1997) foi buscar inspiração na teoria meios-fim (GUTMAN, 1982) para definir valor para o cliente como "a preferência percebida e a avaliação do consumidor dos atributos do produto, do desempenho dos atributos e das consequências resultantes do seu uso que facilitam (ou bloqueiam) o atingimento das metas e propósitos dos consumidores nestas situações de uso". Segundo o autor, esta definição incorpora tanto o valor desejado quanto recebido e enfatiza o valor resultante das percepções, preferências e avaliações percebidas do cliente.

Por sua vez, Holbrook (1999) se baseou na teoria axiológica de valor (HARTMAN, 1973) para definir valor como uma "experiência interativa relativista de preferências". Por experiência, o autor quer dizer que valor reside não apenas na compra mas também na REAd I Porto Alegre - Edição 71 - º 1 - janeiro/abril 2012 - p. 130-160 
experiência que dela deriva o consumidor; interativa significa que valor para o consumidor implica uma interação entre um sujeito (o consumidor) e um objeto (um produto), isto é, o valor depende das características de um objeto físico ou mental mas não ocorre sem o envolvimento de um sujeito que o aprecia; relativista porque o valor para o cliente depende da comparação de diferentes objetos, varia de sujeito para sujeito e depende do contexto em que o julgamento avaliativo ocorre; finalmente, as preferências revelam o significado do julgamento avaliativo. Holbrook (1999) ainda propôs três dimensões (valor extrínseco versus intrínseco, valor orientado para si versus para os outros e valor ativo versus reativo) que, quando combinadas, revelam oito diferentes tipos de valor: eficiência, excelência, status, estima, diversão, estética, ética e espiritualidade.

Embora definições como as de Woodruff (1997) e Holbrook (1999) sejam mais completas e ricas que as definições baseadas na teoria econômica, também é verdade que são muito mais difíceis de se operacionalizar. Em nossa opinião, Sheth et al. (1991) conseguiram oferecer um quadro referencial amplo que combina robustez teórica e viabilidade de operacionalização. Em sua proposta, validada por uma extensa investigação em diversos campos teóricos nos quais o conceito de valor foi examinado, incluindo economia, psicologia social e clínica, antropologia e sociologia, Sheth e seus amigos sugerem que a utilidade percebida de uma escolha, seja no nível da decisão de compra (comprar ou não comprar), no nível do produto (comprar o produto A ou B) ou no nível da marca (comprar a marca A ou B) possui cinco dimensões de valor: social, emocional, funcional, epistêmica e condicional.

O que se percebe é que a pesquisa do consumidor evoluiu de um foco exclusivo nos aspectos cognitivos da tomada de decisão para incluir também aspectos intrínsecos, possibilitando que objetos e experiências possam ser valorizados por si mesmos. Holbrook; Hirschman (1982) argumentaram em favor de uma perspectiva experimental que incluísse os aspectos simbólicos, hedônicos e estéticos do processo de consumo. Outros pesquisadores (BATRA; AHTOLA, 1991) verificaram em sua pesquisa a presença de componentes utilitários e hedônicos distintos, chamados por eles de dimensões de "pensamento e sentimento". Estimulados por estas constatações, Babin et al. (1994) desenvolverem sua escala de valores de compra hedônico e utilitário. Embora reconheçam outras dimensões do valor proporcionado pela experiência de consumo, Babin et al. (1994) justificaram a sua escolha pelo foco nas dimensões hedônica e utilitária em função da sua presença subjacente a diversos fenômenos do consumo e ao desejo de desenvolver uma escala parcimoniosa. 
Valores de compra hedônico e utilitário: os antecedentes e as relações com os resultados do varejo

\subsection{Os valores de compra hedônico e utilitário}

Os consumidores desenvolvem diferentes níveis de valor de compra em função dos produtos e da própria atividade de compra. Segundo Hirschman (1984), entretanto, as constatações das pesquisas tradicionais não refletiam o valor total destas experiências de compra, deixando de reconhecer e medir outros benefícios e inúmeros custos intangíveis e emocionais de uma experiência de compra.

A definição de que o valor de compra é um processo de aquisição funcional e objetivo de produtos é muito limitada para refletir uma experiência completa de compra (HIRSCHMAN; HOLBROOK, 1982). Embora a experiência de compra hedônica seja geralmente considerada mais importante do que a mera aquisição de produtos, o lado festivo, lúdico ou luxuriante da compra tem sido pesquisado com menor frequência (SHERRY, 1990).

Diante disto, diversas pesquisas analisaram a capacidade de uma experiência de compra fornecer valor de compra hedônico. Por exemplo, os consumidores podem usufruir os benefícios dos produtos mesmo sem comprá-los (MACINNIS; PRICE, 1987). A própria satisfação percebida é um importante benefício hedônico de uma experiência de compra (BLOCH; SHERRELL; RIDGWAY, 1986). As compras hedônicas induzem o consumidor à excitação, ao envolvimento, à liberdade, à realização fantasiosa e ao escapismo (HIRSCHMAN, 1983). Para alguns consumidores, as compras hedônicas adquirem as características de uma caçada, enfatizando a perseguição e a descoberta (THALER, 1985). Estes consumidores gastam seus finais de semana procurando ofertas e sentem prazer em obter descontos (BELK; SHERRY; WALLENDORF, 1988). Eles também procuram liquidações, experimentando muitas das mesmas sensações que ocorrem numa caçada (VASKE; FEDLER; GRAFE, 1986).

Por outro lado, o valor de compra utilitário é relevante para os consumidores que buscam o cumprimento de objetivos e riscos mais baixos (BATRA; AHTOLA, 1991). Neste sentido, Babin et al. (1994) relataram que os compradores orientados para o valor utilitário derivam valor de uma experiência de compra somente se ela for completada com sucesso.

A distinção entre a compra hedônica e utilitária fica mais evidente ao se analisar o estudo de Lehtonen (1994 apud Chang, 2001). Segundo o pesquisador, a compra hedônica é um fim em si mesmo e, portanto, não necessariamente inclui a compra. Ela é frequentemente acompanhada por impulsos de compra. Como este tipo de compra busca o prazer, a eficiência não é importante e, portanto, não existe um ponto de início e fim, o que enfatiza o seu aspecto experimental. Por outro lado, a compra utilitária é um meio para um fim e, portanto, necessariamente inclui a compra. Por ser planejada, ela não é acompanhada por impulsos de 
compra. Como este tipo de compra não busca o prazer, a eficiência é importante e, portanto, existe um ponto de início e fim da compra, o que enfatiza o seu aspecto racional.

Assim, a compra hedônica pode desempenhar papéis positivos e negativos em relação aos benefícios proporcionados ao consumidor na experiência de compra. A forma negativa extrema da compra hedônica é a compra impulsiva ou compulsiva. Neste sentido, Rook (1987) ressaltou que os compradores impulsivos compram produtos mais pela necessidade de comprar do que pela real necessidade do produto.

Por outro lado, a compra hedônica pode também trazer contribuições positivas. As compras hedônicas podem ser terapêuticas (BABIN et al., 1994). Muitos consumidores engajam-se na compra hedônica como um meio de gerenciamento do seu humor (BAUMANN; CIALDINI; KENRICK, 1981). Os compradores com fins hedônicos parecem derivar sua gratificação de prazeres hedônicos imediatos (FISCHER; ARNOLD, 1990). A compra hedônica é geralmente expressa pelos aspectos recreacionais da busca de uma loja, independentemente da compra ocorrer (JARBOE; MCDANIEL, 1987). O prazer da compra inclui a oportunidade de interação social com amigos, familiares ou até mesmo com estranhos, além da estimulação sensorial, que inclui o escapismo da rotina da vida diária e novas informações à respeito das tendências e da moda (WESTBROOK; BLACK, 1985).

Embora os consumidores busquem tanto o valor de compra hedônico como o utilitário na maioria das situações de compra, o ato de fazer compras pode ser considerado como fazer compras com um objetivo (compra utilitária) ou fazer compras como um objetivo (compra hedônica).

\subsection{As variáveis ambientais da loja e o valor de compra hedônico}

O estudo realizado por Bloch et al. (1994) sugere uma relação entre as características ambientais da loja e o valor de compra hedônico. Segundo os pesquisadores, o ambiente físico de uma loja, incluindo layout, arquitetura, decoração, iluminação, música, aromas e limpeza, dentre outros, influencia o estado emocional dos consumidores. Neste sentido, sabe-se que a compra hedônica é geralmente expressa pelos aspectos recreacionais da busca de uma loja, independentemente da compra ocorrer. Deste modo, sugere-se que o ambiente de uma loja poderá suprir estes aspectos recreacionais que um consumidor geralmente busca em uma compra hedônica.

Além disso, a emoção positiva suscitada pelo ambiente estimulante de uma loja encoraja o consumidor a permanecer por mais tempo nela e a interagir com outras pessoas (BABIN; DARDEN, 1995; DONOVAN; ROSSITER, 1982). De fato, outros estudos REAd I Porto Alegre - Edição 71 - N 1 - janeiro/abril 2012 - p. 130-160 
Valores de compra hedônico e utilitário: os antecedentes e as relações com os resultados do varejo

destacaram que a compra hedônica pode trazer contribuições positivas, como a oportunidade de interação social com amigos, familiares ou até mesmo com estranhos e a estimulação sensorial, tal como o escapismo da rotina da vida diária e novas informações à respeito das tendências e moda (WESTBROOK; BLACK, 1985). Deste modo, sugere-se que a emoção positiva suscitada pelo ambiente de uma loja estimulará a interação social que um consumidor geralmente busca em uma compra hedônica. Portanto, dadas as razões acima, deveríamos esperar os seguintes relacionamentos:

H1 - As carcaterísticas do ambiente físico de uma loja influenciarão positivamente o valor de compra hedônico.

H2 - Os aspectos de organização de uma loja influenciarão positivamente o valor de compra hedônico.

H3 - O sortimento de produtos de uma loja influenciará positivamente o valor de compra hedônico.

H4 - O atendimento de uma loja influenciará positivamente o valor de compra hedônico.

\subsection{As variáveis ambientais da loja e o valor de compra utilitário}

Diversos estudos indicaram que o sortimento da loja, os preços, a equipe de vendas, o fato de a loja estar ou não muito cheia, e os elementos periféricos dos serviços do varejista, podem também contribuir para a avaliação da natureza de uma compra (JONES, 1999; COTTET et al., 2006). O estudo realizado por Lehtonen (1994 apud Chang, 2001) sugere uma relação entre estas características ambientais da loja e o valor de compra utilitário. Corredores amplos, iluminação clara, prateleiras organizadas, agilidade no atendimento e bom sortimento podem aumentar a conveniência e a rapidez de uma compra, deixando no consumidor a sensação de que ele foi mais eficiente e conseguiu fazer mais em menos tempo. Portanto, sugere-se que as variáveis ambientais da loja também influenciam positivamente o valor de compra utilitário, conduzindo às seguintes hipóteses:

H5 - As características do ambiente físico de uma loja influenciarão positivamente o valor de compra utilitário.

H6 - Os aspectos de organização de uma loja influenciarão positivamente o valor de compra utilitário. 
H7 - O sortimento de produtos de uma loja influenciará positivamente o valor de compra utilitário.

H8 - O atendimento de uma loja influenciará positivamente o valor de compra utilitário.

\subsection{Os valores de compra e a satisfação}

Diversas pesquisas concluíram que os julgamentos de satisfação são formados por elementos afetivos e cognitivos (WESTBROOK, 1987; OLIVER; SWAN, 1989; OLIVER, 1993). Segundo Oliver; Swan (1989), as respostas afetivas surgem da avaliação dos resultados obtidos a partir do uso dos produtos, da interpretação cognitiva e de processos relacionados. Portanto, os julgamentos de satisfação dependem das experiências afetivas acumuladas com o produto e das crenças e outros conhecimentos retidos e atualizados ao longo do tempo.

Oliver (1997) também salientou que os eventos de consumo são capazes de satisfazer necessidades em níveis funcionais e psicológicos. Neste caso, os atributos mais tangíveis dos produtos, por serem bivalentes, contribuem para a satisfação ou insatisfação dos consumidores. Por fornecerem informações aos processos cognitivos, estes atributos devem estar intimamente relacionados às avaliações do valor de compra utilitário. Por outro lado, as características mais abstratas e superiores dos produtos, por serem monovalentes, contribuem de uma maneira afetiva e psicológica para a satisfação dos consumidores. Assim, os atributos monovalentes dos produtos devem estar intimamente relacionados às avaliações do valor de compra hedônico.

Portanto, fica evidente que as percepções de valor em uma experiência de compra são componentes importantes dos julgamentos de satisfação. $\mathrm{Na}$ verdade, diversos estudos mostraram a relação entre os valores de compra hedônico e utilitário e a satisfação (BABIN et al., 1994; BABIN et al., 2005; JONES et al., 2006). Deste modo, deveríamos esperar os seguintes relacionamentos:

\section{H9 - O valor de compra hedônico influenciará positivamente a satisfação com a compra.}

H10 - O valor de compra utilitário influenciará positivamente a satisfação com a compra.

Entretanto, a natureza da experiência de compra sugere que a satisfação deve estar mais fortemente relacionada com o valor de compra hedônico do que com o utilitário. Segundo REAd I Porto Alegre - Edição 71 - N 1 - janeiro/abril 2012 - p. 130-160 
Valores de compra hedônico e utilitário: os antecedentes e as relações com os resultados do varejo

Fournier; Mick (1999), isso acontece porque a satisfação depende do contexto do consumo.

De fato, diversas pesquisas realizadas em contextos hedônicos identificaram relações mais fortes entre as respostas emocionais e a satisfação (OLIVER; RUST; VARKI, 1997; WAKEFIELD; BAKER, 1998). Portanto, tendo em vista a capacidade da compra suscitar respostas emocionais substanciais e o fato das emoções estarem intimamente relacionadas com a satisfação, é razoável esperar que a influência do valor de compra hedônico sobre a satisfação com a compra será maior do que a influência do valor de compra utilitário, o que conduz à seguinte hipótese:

\section{H11 - O valor de compra hedônico terá uma influência maior sobre a satisfação com a compra do que o valor de compra utilitário.}

\subsection{Os valores de compra e o boca a boca}

O boca a boca tem sido encarado como uma fonte confiável e poderosa de informações no contexto do varejo (HIGIE; FEICK; PRICE, 1987). Segundo Swan; Oliver (1989), o boca a boca é uma conseqüência das respostas emocionais do consumidor a situações de consumo. O boca a boca surge do forte envolvimento do consumidor com o produto, que por sua vez cria uma tensão psicológica liberada pelo compartilhamento da experiência com outros (DICHTER, 1966; WESTBROOK, 1987). Deste modo, deveríamos esperar um relacionamento entre o valor de compra hedônico e o boca a boca.

Por outro lado, pesquisas também mostraram que o boca a boca está associado a processos cognitivos, tais como as percepções de valor e as avaliações de equidade (HARTLINE; JONES, 1996). Estes estudos demonstraram que o boca a boca é uma atividade que requer dos consumidores o seu engajamento na análise de atributos, na interpretação de eventos e no compartilhamento de informações com outros. Assim, tendo em vista que o valor de compra utilitário foca nos inputs/outputs de uma experiência de compra, é provável que ele influencie o boca a boca. Deste modo, deveríamos esperar os seguintes relacionamentos:

\section{H12 - O valor de compra hedônico influenciará positivamente o boca a boca positivo.}

H13 - O valor de compra utilitário influenciará positivamente o boca a boca positivo.

Entretanto, evidências sugerem que o valor de compra hedônico está mais fortemente relacionado ao boca a boca do que o valor de compra utilitário. As pessoas consideram os aspectos utilitários como um meio de preservação do status-quo e os aspectos hedônicos REAd I Porto Alegre - Edição 71 - № 1 - janeiro/abril 2012 - p. 130-160 
como um meio para se intensificar a experiência do consumo (KAHN; MEYER, 1991). Assim, compartilhar com outros os aspectos de uma experiência de compra pode ser visto como um meio para intensificá-la, aumentando a gratificação e melhorando a autoestima do consumidor. De fato, quando a experiência de compra é excepcionalmente prazerosa, as pessoas são motivadas a compartilhar suas experiências com outros e a encorajar seus amigos e familiares a tornarem-se clientes de um determinado varejista (HIGIE et al., 1987). Portanto, a seguinte hipótese pode ser formulada:

\section{H14 - O valor de compra hedônico terá uma influência maior sobre o boca a boca positivo do que o valor de compra utilitário.}

\subsection{Os valores de compra e a intenção de recompra}

A intenção de recompra reflete a probabilidade de um cliente comprar novamente em uma loja de varejo (OLIVER, 1999). O estudo conduzido por Wakefield; Barnes (1996) sugere uma ligação entre os valores de compra hedônico e utilitário e a intenção de recompra.

De fato, estudos na área de psicologia mostraram que as experiências afetivas são importantes antecedentes das motivações de aproximação ou de afastamento, tais como a intenção de recompra (DONOVAN; ROSSITER, 1982). Portanto, deve-se esperar que o valor de compra hedônico esteja relacionado com a intenção de recompra.

Por outro lado, o valor de compra utilitário também está relacionado com a intenção de recompra. A percepção de valor de compra utilitário em uma experiência de consumo provavelmente suscitará no consumidor a sensação de que ele completou com sucesso a aquisição do produto. Além disso, constatou-se que consumidores que percebem maior valor utilitário desenvolvem uma maior percepção da qualidade do produto e da própria experiência de compra, exibindo mais fortemente a intenção de recompra (BABIN; BABIN, 2001). Portanto, dadas as razões acima, deveríamos esperar os seguintes relacionamentos:

\section{H15 - O valor de compra hedônico influenciará positivamente a intenção de recompra. H16 - O valor de compra utilitário influenciará positivamente a intenção de recompra.}

O valor de compra utilitário está mais fortemente relacionado com a intenção de recompra do que o valor de compra hedônico. $\mathrm{Na}$ verdade, quando os consumidores são confrontados com alternativas opostas, eles buscam razões e argumentos para justificar sua escolha (DHAR; WERTENBROCH, 2000). Nestas situações de decisão, que estimulam a 
Valores de compra hedônico e utilitário: os antecedentes e as relações com os resultados do varejo

racionalização, provavelmente os aspectos utilitários da compra serão enfatizados (TVERSKY; SATTAH; SLOVIC, 1988). De fato, a intenção de recompra pode ser análoga a uma situação de escolha na qual o consumidor precisa de mais justificativas para o alcance de um objetivo que requer esforço.

Portanto, os consumidores enfatizarão o valor de compra utilitário quando estiverem avaliando a possibilidade de visitar novamente um varejista. Assim, deveríamos esperar que o valor de compra utilitário esteja mais fortemente relacionado com a intenção de recompra do que o valor de compra hedônico. Portanto, a seguinte hipótese pode ser formulada:

\section{H17 - O valor de compra utilitário terá uma influência maior sobre a intenção de recompra do que o valor de compra hedônico.}

As hipóteses formuladas nas seções anteriores estão consolidadas no modelo teórico representado na figura 1. A partir deste modelo, se pode inferir os seguintes relacionamentos:

- os antecedentes dos valores de compra hedônico e utilitário são as variáveis ambientais da loja - o ambiente físico, a organização, o sortimento e o atendimento;

- os conseqüentes ou resultados dos valores de compra hedônico e utilitário são as variáveis de resultado da loja - a satisfação, o boca a boca e a intenção de recompra;

- o ambiente físico, a organização, o sortimento e o atendimento influenciam os valores de compra hedônico e utilitário;

- os valores de compra hedônico e utilitário influenciam a satisfação, o boca a boca e a intenção de recompra. 


\section{João Marques Teixeira \& José Mauro da Costa Hernandez}

Figura 1: Modelo estrutural de relacionamentos

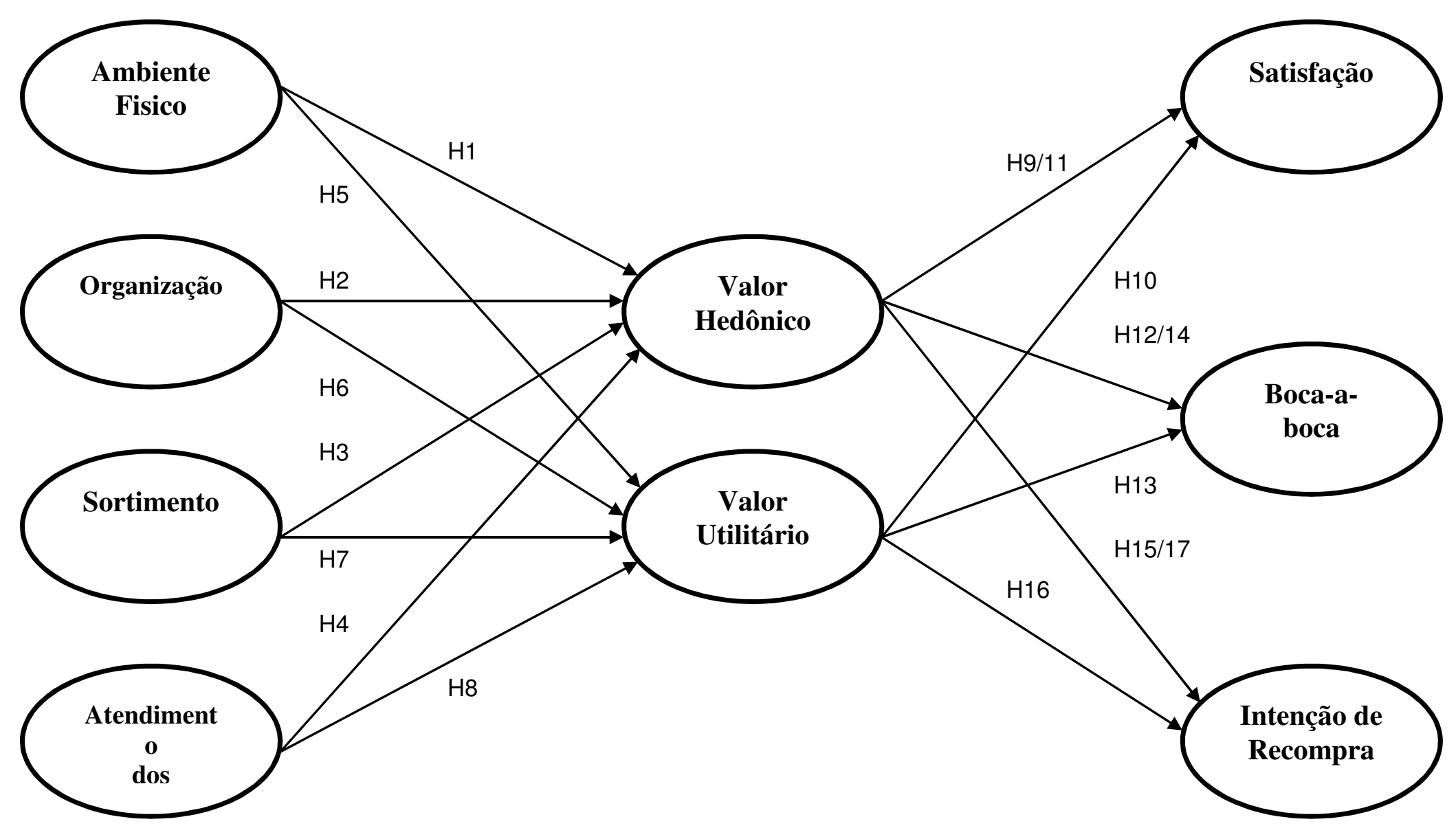

Fonte: Autor 
Valores de compra hedônico e utilitário: os antecedentes e as relações com os resultados do varejo

\section{MÉTODO}

Esta seção apresenta os procedimentos metodológicos que levaram ao teste dos relacionamentos apresentados no modelo teórico (Figura 1). Os dados foram obtidos por meio de um levantamento transversal e as hipóteses do modelo teórico foram testadas por meio de modelagem de equações estruturais. A seguir detalhamos os procedimentos metodológicos adotados no estudo.

\subsection{Amostra}

Os dados foram coletados em lojas de uma grande rede varejista brasileira que comercializa eletrodomésticos e vestuário. Limitamos a coleta de dados apenas aos indivíduos que haviam acabado de fazer uma compra de eletrodomésticos de valor superior a $\mathrm{R} \$ 500,00$. Com esta decisão, optamos por incluir na amostra apenas compras de uma única grande categoria de produtos para evitar eventuais diferenças na avaliação de produtos tão diferentes quanto vestuário e eletrodomésticos. Também acreditamos que compras de menor valor não geram experiências memoráveis em termos de valor hedônico e daí a decisão de limitarmos as compras a valores superiores a $\mathrm{R} \$ 500,00$.

As entrevistas foram realizadas em quatro lojas localizadas na cidade de São Paulo. Os indivíduos foram selecionados por meio de um processo de amostragem não probabilístico por conveniência.

\subsection{Procedimentos}

Dois entrevistadores profissionais contratados para coletar os dados desta pesquisa ficaram nas quatro lojas durante quatro finais de semana (sexta-feira a domingo), dias de maior movimento de compradores na seção de eletrodomésticos. A autorização para a coleta de dados foi obtida previamente do diretor regional de lojas deste varejista e os gerentes da loja foram comunicados sobre a presença dos entrevistadores.

Durante a coleta, os entrevistadores se posicionaram próximo ao caixa de pagamentos ou à seção de crédito ao consumidor. Os clientes eram abordados enquanto faziam o pagamento ou enquanto esperavam que suas solicitações de crédito fossem autorizadas.

\subsection{Instrumento de coleta de dados}

Inicialmente, os respondentes foram solicitados a proporcionar detalhes sobre a compra efetuada naquele dia como o tipo, a marca, o modelo e o preço do produto comprado, bem como a forma de pagamento utilizada. Também foi perguntado se o respondente possuía o cartão da loja e se o produto havia sido comprado para presente ou para si mesmo.

A seguir, foi mensurada a satisfação com a compra utilizando quatro itens da escala adaptada de Maxhan; Netemeyer (2002). Em seguida, os respondentes proporcionaram 
respostas para as escalas de valor de compra hedônico (sete itens) e utilitário (seis itens) de Babin et al. (1994). No passo seguinte, os respondentes foram solicitados a avaliar 14 itens do ambiente da loja numa escala de onze pontos (zero a dez). Os itens foram adaptados de diversas escalas de imagem de loja (MARTINEAU, 1958; LINDQUIST, 1974; MAZURSKY; JACOBY, 1986) e que, em nossa opinião, faziam mais sentido para este varejista em particular e se relacionavam mais estreitamente ao conceito de ambiente físico de uma loja. Em seguida, foi mensurada a intenção de recompra utilizando três itens adaptados de Mittal; Ross; Baldasare (1998) e a intenção de boca a boca utilizando três itens adaptados de Maxham; Netemeyer (2002). Todos os itens foram mensurados por escalas do tipo Likert com cinco pontos e encontram-se descritos no Anexo 1.

Finalmente, também foram solicitadas informações demográficas (sexo, estado civil, idade, escolaridade e renda).

\subsection{Análise dos Dados}

Para a análise dos dados foram utilizados os softwares Statistical Package for Social Science (SPSS) 13.0 e LISREL 8.51. As estatísticas descritivas foram utilizadas para a apresentação do perfil demográfico dos respondentes. A análise fatorial exploratória foi utilizada para a avaliação da validade convergente e discriminante do modelo de mensuração. Foram analisadas as comunalidades extraídas e as cargas fatoriais dos indicadores e foram realizados os testes de esfericidade (qui-quadrado) e de Kaiser-Meyer-Olkin. O teste de consistência interna utilizou o modelo alpha de Cronbach. A análise fatorial confirmatória foi utilizada para a avaliação do modelo estrutural.

\section{RESULTADOS}

A amostra foi composta por 399 respondentes, sendo que $46 \%$ dos entrevistados eram homens e 55\% eram casados. Os respondentes com idade entre 21 e 45 anos representaram $73 \%$ da amostra. O nível de escolaridade predominante foi o colegial (50\%), seguido pelo nível superior (22\%). A grande maioria (79\%) possuía renda entre $\mathrm{R} \$ 501,00$ e $\mathrm{R} \$ 3.000,00$.

Inicialmente, realizamos uma análise fatorial exploratória (via componentes principais com rotação varimax) dos 3 grupos de variáveis (ambiente da loja, valores hedônico e utilitário e variáveis de resultado) já que elas de fato compõem grupos distintos de variáveis.

$\mathrm{Na}$ primeira tentativa de análise fatorial exploratória com as quatro variáveis latentes do ambiente da loja, a comunalidade extraída dos indicadores NTEM e NIDE foi menor que 0,5 e o indicador NLIM tinha com carga fatorial cruzada superior a 0,4. Deste modo, estes três indicadores foram eliminados das análises subsequentes. $\mathrm{Na}$ segunda tentativa, a 
Valores de compra hedônico e utilitário: os antecedentes e as relações com os resultados do varejo

comunalidade extraída de todos os indicadores foi maior que 0,5 , o teste de esfericidade de Bartlett foi significativo ao nível de $0,1 \%$, o resultado do teste Kaiser-Meyer-Olkin foi maior que 0,7 e o coeficiente de consistência interna Alfa de Cronbach foi superior a 0,7. A variância explicada foi de $21 \%$ para o ambiente físico, $18 \%$ para a organização, $18 \%$ para o sortimento e $22 \%$ para o atendimento dos vendedores.

A seguir, passamos para a análise conjunta dos indicadores das duas variáveis latentes de valores de compra hedônico e utilitário. Na primeira tentativa, a comunalidade extraída do indicador VU5 foi menor que 0,5 e os indicadores VH2, VH7, VU1 e VU4 tinham carga fatorial cruzada superior a 0,4 . Deste modo, estes cinco indicadores foram eliminados das análises subsequentes. Na segunda tentativa, a comunalidade extraída de todos os indicadores foi superior a 0,5, o teste de esfericidade de Bartlett foi significativo ao nível de 0,1\%, o resultado do teste de Kaiser-Meyer-Olkin foi maior que 0,7 e o coeficiente de consistência interna Alfa de Cronbach foi maior que 0,7. A variância explicada foi de $40 \%$ para o valor de compra hedônico e $26 \%$ para o valor de compra utilitário.

$\mathrm{Na}$ única tentativa com os indicadores das três variáveis latentes de resultados do varejo, todos os indicadores tiveram comunalidade extraída maior que 0,5 , o teste de esfericidade de Bartlett foi significativo ao nível de $0,1 \%$, o resultado do teste de Kaiser-Meyer-Olkin foi maior que 0,7 e o coeficiente de consistência interna Alfa de Cronbach foi superior a 0,7. A variância explicada foi de $33 \%$ para a variável satisfação com a compra, $29 \%$ para a intenção de boca a boca e $23 \%$ para a intenção de recompra. Deste modo, os resultados da análise do modelo de mensuração indicam que o modelo possui validade convergente e discriminante e que as escalas utilizadas têm confiabilidade aceitável.

O próximo passo foi testar o modelo estrutural e mais dois modelos alternativos. Além de testar as hipóteses de relacionamentos expressas pelo modelo estrutural, a análise também tinha como objetivo verificar se os valores de compra hedônico e utilitário exerciam o papel de intermediação entre as variáveis ambientais da loja e as variáveis de resultado do varejo.

$\mathrm{Na}$ primeira tentativa com o modelo estrutural proposto, após examinar-se os valores padronizados e os índices de ajustamento, percebeu-se que 7 caminhos não significativos deveriam ser eliminados. Os resultados da segunda tentativa encontram-se nas tabelas 1 e 2 e estão representados graficamente no modelo estrutural da figura 2.

Os resultados indicam que o ambiente físico, a organização e o sortimento de uma loja não se associaram de nenhuma forma com os valores de compra hedônico e utilitário. Além disso, o valor de compra utilitário não se associou com o boca a boca e associou-se negativamente com a satisfação e com a intenção de recompra. Por outro lado, o atendimento 
se associou com os valores de compra hedônico e utilitário. Finalmente, o valor de compra hedônico se associou com as três variáveis de resultado do varejo - a satisfação, o boca a boca e a intenção de recompra.

Tabela 1: Valores padronizados do modelo estrutural final

\begin{tabular}{|c|c|c|c|c|}
\hline Hip. & Caminho & $\begin{array}{c}\text { Valor } \\
\text { Padronizado } \\
\end{array}$ & $Z$ & Conclusão \\
\hline H1 & Ambiente Físico $\rightarrow$ Valor de compra hedônico & n.s. & & Rejeitada \\
\hline $\mathrm{H} 2$ & Organização $\rightarrow$ Valor de compra hedônico & n.s. & & Rejeitada \\
\hline $\mathrm{H} 3$ & Sortimento $\rightarrow$ Valor de compra hedônico & n.s. & & Rejeitada \\
\hline $\mathrm{H} 4$ & $\begin{array}{l}\text { Atendimento dos Vendedores } \rightarrow \text { Valor de compra } \\
\text { hedônico }\end{array}$ & 0,44 & $7,26^{* * * *}$ & Não Rejeitada \\
\hline H5 & Ambiente Físico $\rightarrow$ Valor de compra utilitário & n.s. & & Rejeitada \\
\hline H6 & Organização $\rightarrow$ Valor de compra utilitário & n.s. & & Rejeitada \\
\hline $\mathrm{H7}$ & Sortimento $\rightarrow$ Valor de compra utilitário & n.s. & & Rejeitada \\
\hline $\mathrm{H} 8$ & $\begin{array}{l}\text { Atendimento dos Vendedores } \rightarrow \text { Valor de compra } \\
\text { utilitário }\end{array}$ & 0,62 & $7,85^{* * * *}$ & Não Rejeitada \\
\hline H9 & Valor de compra hedônico $\rightarrow$ Satisfação & 2,52 & $4,05^{* * * *}$ & Não Rejeitada \\
\hline $\mathrm{H} 10$ & Valor de compra utilitário $\rightarrow$ Satisfação & $-2,07$ & $-3,41^{* * * *}$ & Rejeitada \\
\hline $\mathrm{H} 12$ & Valor de compra hedônico $\rightarrow$ Boca a boca & 0,60 & $10,18^{* * * *}$ & Não Rejeitada \\
\hline H13 & Valor de compra utilitário $\rightarrow$ Boca a boca & n.s. & & Rejeitada \\
\hline H15 & Valor de compra hedônico $\rightarrow$ Intenção de recompra & 1,38 & $4,58^{* * * *}$ & Não Rejeitada \\
\hline H16 & Valor de compra utilitário $\rightarrow$ Intenção de recompra & $-0,82$ & $-2,85^{* * *}$ & Rejeitada \\
\hline \multicolumn{5}{|c|}{$\mathrm{RMSEA}=0,053 ; \mathrm{NFI}=0,91 ; \mathrm{CFI}=0,95 ; \mathrm{RMR}=0,063 ; \mathrm{GFI}=0,90$} \\
\hline \multirow{2}{*}{\multicolumn{5}{|c|}{ Fonte: autor }} \\
\hline & & & & \\
\hline \multicolumn{5}{|c|}{ Tabela 2: $r^{2}$ das variáveis latentes do modelo estrutural final } \\
\hline \multicolumn{3}{|c|}{ Variáveis de resultado } & & $r^{2}$ \\
\hline \multicolumn{3}{|c|}{ Satisfação } & & 0,55 \\
\hline \multicolumn{3}{|c|}{ Boca a boca } & & 0,36 \\
\hline \multicolumn{3}{|c|}{ Intenção de recompra } & & 0,39 \\
\hline \multicolumn{3}{|c|}{ Valor de compra hedônico } & & 0,20 \\
\hline \multicolumn{3}{|c|}{ Valor de compra utilitário } & & 0,39 \\
\hline
\end{tabular}

Fonte: autor

Em seguida, foram testados dois modelos alternativos, não mostrados aqui em função da limitação de espaço. A idéia subjacente aos dois modelos alternativos é a mesma, ou seja, testar se os valores de compra hedônico e utilitário intermedeiam a relação entre as variáveis do ambiente da loja e as variáveis de resultado do varejo. Para tanto, nos dois modelos alternativos esta relação de intermediação foi eliminada. Especificamente, no primeiro modelo alternativo, os valores de compra hedônico e utilitário continuaram sendo influenciados pelas variáveis ambientais da loja, mas não influenciavam as variáveis de resultado do varejo, ou seja, os valores de compra hedônico e utilitário deixaram de intermediar a relação e passaram a ser variáveis de resultado. No segundo modelo alternativo, os valores de compra hedônico e utilitário continuaram influenciando as variáveis de 
Valores de compra hedônico e utilitário: os antecedentes e as relações com os resultados do varejo

resultado do varejo mas não eram influenciadas pelas variáveis do ambiente da loja, isto é, os valores de compra hedônico e utilitário deixaram de intermediar a relação e passaram a ser variáveis antecedentes.

$\mathrm{Na}$ primeira tentativa de análise do primeiro modelo alternativo, após examinar os valores padronizados e os índices de ajustamento, percebeu-se que 11 caminhos não significativos deveriam ser eliminados. Na segunda tentativa, eliminando-se os caminhos rejeitados, os resultados indicaram um bom ajuste do modelo $(\mathrm{RMSEA}=0,053, \mathrm{NFI}=0,92$, $\mathrm{CFI}=0,95, \mathrm{RMR}=0,051$ e GFI $=0,90)$. Os coeficientes de explicação para cada uma das variáveis de resultado foram 0,04 para a satisfação, 0,11 para o boca a boca, 0,04 para a intenção de recompra, 0,19 para o valor de compra hedônico e 0,32 para o valor de compra utlitário. Apesar dos bons índices de ajuste, os coeficientes de explicação do primeiro modelo alternativo se mostraram inferiores àqueles obtidos com o modelo proposto originalmente com intermediação dos valores de compra utilitário e hedônico.

$\mathrm{Na}$ primeira tentativa de análise do segundo modelo alternativo, após o exame dos valores padronizados e dos índices de ajustamento, percebeu-se que 11 caminhos não significativos deveriam ser eliminados. Eliminados os caminhos, na segunda tentativa os resultados indicaram um bom ajuste do modelo (RMSEA $=0,052, \mathrm{NFI}=0,92, \mathrm{CFI}=0,95$, RMR $=0,049$ e GFI = 0,90). Os coeficientes de explicação para cada uma das variáveis de resultado foram 0,46 para a satisfação, 0,38 para o boca a boca e 0,41 para a intenção de recompra. Embora os coeficientes de explicação sejam ligeiramente inferiores aos proporcionados pelos coeficientes do modelo proposto original, os coeficientes de explicação das variáveis de resultado são muito próximos aos valores obitdos no modelo original. Portanto, estes resultados lançam dúvidas se os valores de compra hedônico e utilitários se mostraram boas variáveis intermediadoras entre as variáveis do ambiente da loja e as variáveis de resultado do varejo. 


\section{João Marques Teixeira \& José Mauro da Costa Hernandez}

Figura 2: Os valores padronizados do modelo estrutural

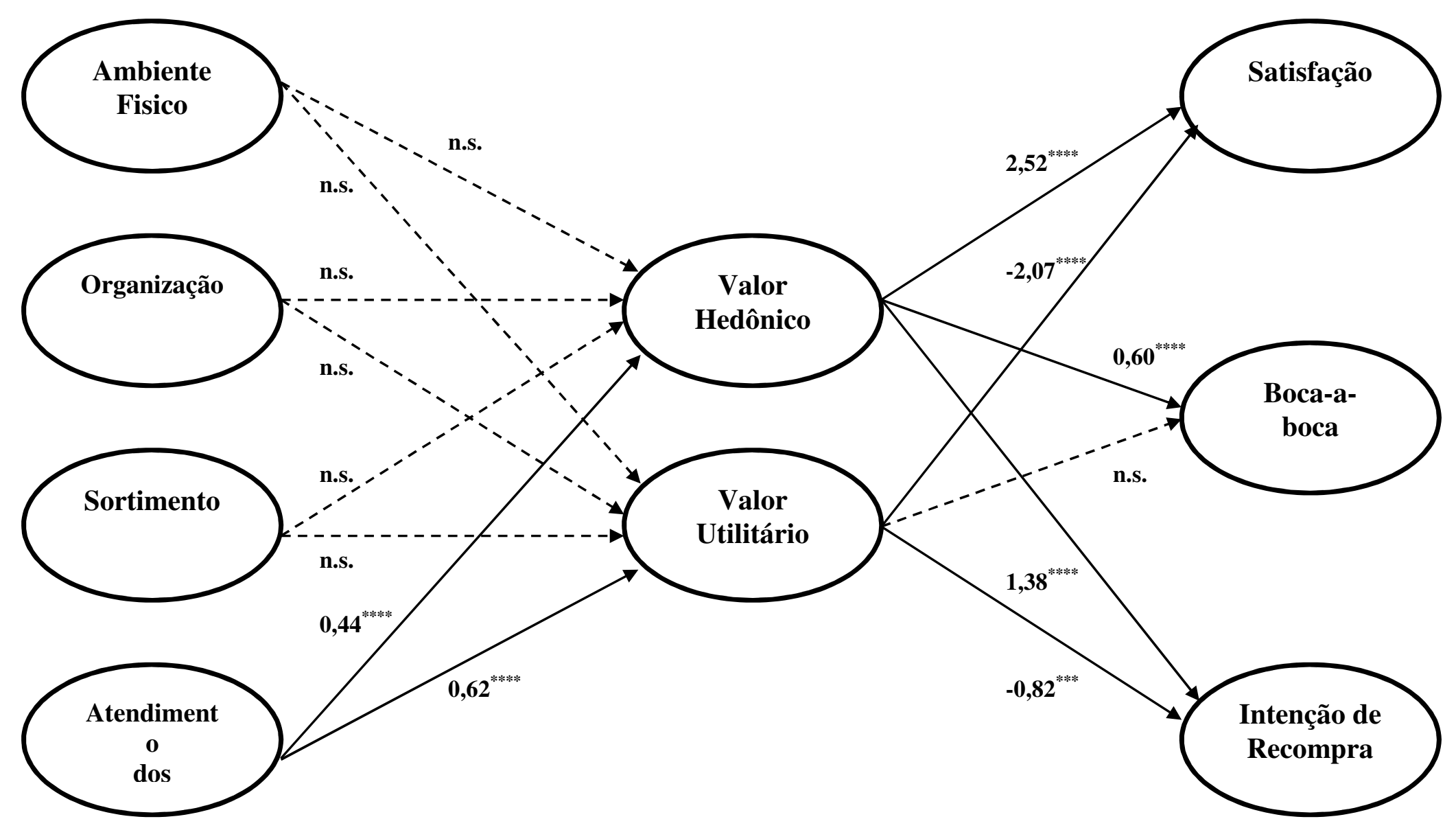

Fonte: autor 
Valores de compra hedônico e utilitário: os antecedentes e as relações com os resultados do varejo

\section{COMENTÁRIOS FINAIS}

Ao contrário do que era esperado, três das variáveis ambientais da loja (ambiente físico, organização e sortimento) não influenciaram os valores de compra hedônico e utilitário. Uma das possíveis explicações para este resultado é que as lojas onde os dados foram coletados não possuem um ambiente que estimule os consumidores. Por se tratar de uma rede de varejo mais voltada para as camadas populares, as lojas desta rede de varejo possuem uma arquitetura comum, decoração simples, layout extremamente otimizado e uma variedade pequena de marcas e produtos.

Além desta limitação, também notamos problemas na escala de valor utilitário. Durante a análise fatorial, três indicadores do valor de compra utilitário foram eliminados em função da baixa comunalidade extraída e da elevada carga fatorial cruzada, restando apenas três indicadores que se concentraram mais em torno do fator tempo. Deste modo, é provável que esta limitação da escala tenha se refletido nas relações envolvendo o valor de compra utilitário.

Como havia sido previsto, os resultados demonstraram que o atendimento dos vendedores influenciou positivamente os valores de compra hedônico e utilitário, corroborando os estudos de Jones (1999) e Cottet et al. (2006). Uma análise complementar dos indicadores do valor de compra hedônico indica que a agradabilidade seja um aspecto central desta variável. Assim, sugere-se que um bom atendimento tenha contribuído para a criação de um ambiente agradável de compras. Por outro lado, a compra utilitária se caracteriza pelos seus aspectos racionais, tais como o cumprimento de objetivos, o planejamento e a eficiência. Da mesma forma, uma análise complementar dos indicadores do valor de compra utilitário indica que a eficiência seja um aspecto central desta variável latente. Assim, sugere-se que a rapidez no atendimento tenha contribuído para a avaliação positiva do consumidor sobre sua eficiência na compra.

Como havia sido previsto, os resultados demonstraram que o valor de compra hedônico influenciou positivamente a satisfação, o boca a boca e a intenção de recompra. Além disso, o valor de compra hedônico exerceu maior influência sobre a satisfação e o boca a boca do que o valor de compra utilitário. Estes resultados estão em linha com aqueles obtidos por Babin et al. (2005) e de Jones et al. (2006).

Ao contrário do que era esperado, os resultados demonstraram que o valor de compra utilitário não influenciou positivamente a satisfação, o boca a boca e a intenção de recompra. Além disso, o valor de compra utilitário também não exerceu maior influência sobre a intenção de recompra do que o valor de compra hedônico. Uma das possíveis explicações é 
que a compra de eletrodomésticos possa ser mais hedônica do que utilitária, diminuindo a influência do valor de compra utilitário. Segundo Dhar; Wertenbroch (2000), os consumidores consideram os produtos utilitários como um meio de preservação dos benefícios no dia-a-dia e os produtos hedônicos como uma ferramenta de realce da qualidade de vida. A análise dos dados sugere que aproximadamente $50 \%$ dos produtos comprados poderiam ser classificados como produtos primariamente hedônicos. Deste modo, é provável que o foco da pesquisa em compras de eletrodomésticos possa ter influenciado os níveis de valor de compra utilitário derivados pelos consumidores no evento da compra.

De forma combinada, os resultados trazem tanto contribuições teóricas, gerenciais e metodológicas. Do ponto de vista teórico, estes resultados contribuem para a continuidade do debate sobre os relacionamentos entre as variáveis ambientais da loja, os valores de compra e as variáveis de resultado do varejo. Além disso, o estudo de Jones et al. (2006) foi parcialmente replicado em outra região geográfica, em outro segmento do varejo e com outros tipos de produtos, o que permitirá um melhor entendimento sobre estes importantes relacionamentos em outra realidade.

Do ponto de vista metodológico, a análise do modelo de mensuração e do modelo estrutural foi realizada por meio da modelagem de equações estruturais. No estudo de Jones et al. (2006), os autores utilizaram o modelo de equações estruturais apenas para a análise do modelo de mensuração e em seguida, a regressão linear múltipla para a análise do modelo estrutural. Além disso, foram testadas outras escalas para a mensuração dos valores de compra e das variáveis de resultado do varejo.

Do ponto de vista gerencial, estes resultados indicam aos administradores de lojas de varejo que a percepção de valor para o cliente é fortemente influenciada pela presteza dos vendedores e pela rapidez no atendimento. Além disso, estes resultados indicam aos administradores de lojas de varejo que as variáveis críticas de resultado - a satisfação, o boca a boca e a intenção de recompra - foram mais influenciadas pelos aspectos hedônicos do que pelos aspectos utilitários. Assim, pode-se concluir que, ter boa variedade, sortimento, pronta disponibilidade de mercadorias, boa localização, horários adequados e preços competitivos, não é uma garantia para se ter clientes mais satisfeitos e leais.

Entretanto, foram encontradas algumas importantes limitações. O fato das lojas desta rede de varejo possuírem uma arquitetura comum, uma decoração simples, um layout extremamente otimizado e uma variedade pequena de marcas e produtos provavelmente influenciou as avaliações das variáveis ambientais da loja, diminuindo sua influência sobre os valores de compra. Além disso, a maioria dos respondentes demonstrou impaciência diante do REAd I Porto Alegre - Edição 71 - º 1 - janeiro/abril 2012 - p. 130-160 
Valores de compra hedônico e utilitário: os antecedentes e as relações com os resultados do varejo

elevado número de questões a serem respondidas, o que pode ter criado um viés nas avaliações dos respondentes. Deste modo, é provável que este viés tenha contribuído para a ocorrência das cargas fatoriais cruzadas na escala de valor de compra utilitário, o que por sua vez influenciou a avaliação de valor de compra utilitário. Diante disto, as novas pesquisas a serem conduzidos nesta direção deveriam ser realizadas em ambientes com maior apelo sensorial.

Além disso, é provável que a compra de determinados tipos de eletrodomésticos seja mais hedônica do que utilitária. De fato, os resultados indicaram que uma parcela significativa dos produtos comprados pelos respondentes podem ser classificados como produtos primariamente hedônicos. Deste modo, é provável que o foco da pesquisa em compras de eletrodomésticos possa ter influenciado as avaliações de valor de compra utilitário. Diante disto, as novas pesquisas a serem conduzidos nesta direção deveriam ser realizadas com outros tipos de produtos ou amostras.

Os resultados deste estudo também indicam que os valores de compra não exerceram um papel de intermediação significativo entre as variáveis ambientais da loja e as variáveis de resultado do varejo. Provavelmente os valores de compra hedônico e utilitário não sejam tão relevantes em determinados ambientes e situações como afirma a literatura. Deste modo, fica evidente que serão necessários novos estudos nesta área para uma melhor compreensão destes relacionamentos.

\section{REFERÊNCIAS}

BABIN, B. J.; BABIN, L. Seeking something different? A model of schema typicality, consumer affect, purchase intentions and perceived shopping value. Journal of Business Research, Amsterdan, v. 54, n. 2, p. 89-96, 2001.

BABIN, B. J.; DARDEN, W. R. Consumer self-regulation in a retail environment. Journal of Retailing, Amsterdan, v. 71, n. 1, p. 47-70, 1995.

BABIN, B. J.; DARDEN, W. R. Good and bad shopping vibes: spending and patronage satisfaction. Journal of Business Research, Amsterdan, v. 35, n. 3, p. 201-206, 1996.

BABIN, B. J.; DARDEN, W. R.; GRIFFIN, M. Work and/or fun: measuring hedonic and utilitarian shopping value. Journal of Consumer Research, Chicago, v. 20, n. 4, p. 644-656, 1994. 
João Marques Teixeira \& José Mauro da Costa Hernandez

BABIN, B. J. et al. Modeling consumer satisfaction and word-of-mouth: restaurant patronage in Korea. Journal of Services Marketing, Bingley, v. 19, n. 3, p. 133-139, 2005.

BATRA, R.; AHTOLA, O. Measuring the hedonic and utilitarian sources of consumer attitudes. Marketing Letters, New York, v. 2, n. 2, p. 159-170, 1991.

BAUMANN, D. J.; CIALDINI, R. B.; KENRICK, D. T. Altruism as hedonism: helping and self-gratification as equivalents responses. Journal of Personality and Social Psychology, Washington, DC, v. 40, n. 6, p. 1039-1046, 1981.

BELK, R. W. Situational variables and consumer behavior. Journal of Consumer Research, Chicago, v. 2, n. 3, p. 157-164, 1975.

BELK, R. W.; SHERRY, J. F.; WALLENDORF, M. A naturalistic inquiry into buyer and seller behavior at a swap meet. Journal of Consumer Research, Chicago, v. 14, p. 449-470, 1988.

BLOCK, P. H.; RIDGWAY, N. M.; DAWSON, S. A. The shopping mall as consumer habitat. Journal of Retailing, Amsterdan, v. 70, n. 1, p. 23-42, 1994.

BLOCH, P. H.; SHERRELL, D. L.; RIDGWAY, N. M. Consumer search: an extended framework. Journal of Consumer Research, Chicago, v. 13, n. 1, p. 119-126, 1986.

CHANG, E. The mediating role of hedonic shopping value in apparel shopping satisfaction. 2001. 195 p. Tese (Doctor of Philosophy), Oregon State University, Corvallis, 2001.

COTTET, P.; LICHTLÉ, M.C.; PLICHON, V. The role of value in services: A study in a retail environment. The Journal of Consumer Marketing, Bingley, v. 23, n. 4, p. 219-227, 2006.

CRONIN, J. J.; BRADY, M. K.; HULT, G. T. Assessing the effects of quality, value, and customer satisfaction on consumer behavioral intentions in service environments. Journal of Retailing, Amsterdan, v. 2, n. 76, p. 193-218, 2000. 
Valores de compra hedônico e utilitário: os antecedentes e as relações com os resultados do varejo

DHAR, R.; WERTENBROCH, K. Consumer choice between hedonic and utilitarian goods. Journal of Marketing Research, Chicago, v. 37, n. 1, p. 60-71, 2000.

DICHTER, E. A. How word-of-mouth advertising works. Harvard Business Review, Boston, v. 44, p. 147-157, 1966.

DONOVAN, R. J.; ROSSITER, J. R. Store atmosphere: an environmental psychology approach. Journal of Retailing, Amsterdan, v. 58, n. 1, p. 34-57, 1982.

FISCHER, E.; ARNOLD, S. J. More than a labor of love: gender roles and Christmas shopping. Journal of Consumer Research, Chicago, v. 17, n. 3, p. 333 - 345, 1990.

FOURNIER, S.; MICK, D. G. Rediscovering satisfaction. Journal of Marketing, Chicago, v. 63, n. 4, p. 5-23, 1999.

GRIFFIN, M.; BABIN, B. J.; MODIANOS, D. Shopping values of Russian consumers: the impact of habituation in a developing economy. Journal of Retailing, Amsterdan, v. 76, n. 1, p. 33-52, 2000.

GUTMAN, J. A means-end chian model based on consumer categorization processes. Journal of Marketing, Chicago, v. 46, n. 1, p. 60-72, 1982.

HARTLINE, M. D.; JONES, K. C. Employee performance cues in a hotel service environment: influence on perceived service quality, value, word of mouth intentions. Journal of Business Research, Amsterdan, v. 35, n. 3, p. 207-216, 1996.

HARMAN, R.S. The Hartman Value Profile (HVP): Manual of intepretation. Muskegon: Research Concepts, 1973.

HIGIE, R. A.; FEICK, L. F.; PRICE, L. L. Types and amount of word-of-mouth communications about retailers. Journal of Retailing, Amsterdan, v. 63, n. 3, p. 260-278, 1987. 
HIRSCHMAN, E. C. Predictors of self-projection: fantasy fulfillment and escapism. Journal of Social Psychology, Brighton, v. 120, n. 1, p. 63-76, 1983.

HIRSCHMAN, E. C. Experience seeking: a subjectivist perspective of consumption. Journal of Business Research, Amsterdan, v. 12, n. 1, p. 115-136, 1984.

HIRSCHMAN, E. C.; HOLBROOK, M. B. Hedonic consumption: emerging concepts, methods and propositions. Journal of Marketing, Chicago, v. 46, n. 3, p. 92-101, 1982.

HOLBROOK, M.B. Introduction to consumer value. In: HOLBROOK, M.B. (ed.), Consumer value: A framework for analysis and research, p. 1-28. Londres: Routledge, 1999.

HOLBROOK, M. B.; HIRSCHMAN, E. C. The experiential aspects of consumption: consumer fantasies, feelings, and fun. Journal of Consumer Research, Chicago, v. 9, n. 2, p. 132-140, 1982.

HUI, M. K.; BATESON, J. E. Perceived control and consumer choice on the service experience. Journal of Consumer Research, Chicago, v. 18, n. 2, p. 174-185, 1991.

JARBOE, G. R.; MCDANIEL, C. D. A profile of browsers in regional shopping malls. Journal of the Academy of Marketing Science, New York, v. 15, n. 1, p. 46-53, 1987.

JONES, M. Entertaining shopping experience: An exploratory investigation. Journal of Retailing and Consumer Services, Amsterdan, v. 6, n. 3, p. 129-139, 1999.

JONES, M. A; REYNOLDS, K. E.; ARNOLD, M. J. Hedonic and utilitarian shopping value: Investigating differential effects on retail outcomes. Journal of Business Research, Amsterdan, v. 59, n. 9, p. 974-981, 2006.

KAHN, B.; MEYER, R. J. Consumer multi-attribute judgments under attribute-weight uncertainty. Journal of Consumer Research, Chicago, v. 17, n. 4, p. 508-522, 1991. 
Valores de compra hedônico e utilitário: os antecedentes e as relações com os resultados do varejo

LEHTONEN, T. Shoppailu sociaalisena muotona. Sosiologia, v. 3, p. 192-203, 1994.

LEMON, K. N.; WHITE, T. B.; WINER, R. S. Dynamic customer relationship management: incorporating future considerations into the service retention decision. Journal of Marketing, Chicago, v. 66, n. 1, p. 1-14, 2002.

LINDQUIST, J. D. Meaning of image. Journal of Retailing, Amsterdan, v. 50, p. 29-38, 1974.

MACINNIS, D. J.; PRICE, L. L. The role of imagery in information processing: review and extensions. Journal of Consumer Research, Chicago, v. 13, n. 4, p. 473-491, 1987.

MANO, H.; OLIVER, R. L. Assessing the dimensionality and structure of the consumption experience: evaluation, feeling, and satisfaction. Journal of Consumer Research, Chicago, v. 20, n. 3, p. 451-466, 1993.

MARTINEAU, P. D. The personality of the retail store. Harvard Business Review, Boston, v.36, p. 47-55, 1958.

MATHWICK, C.; MALHOTRA, N.; RIGDON, E. Experiential value: Conceptualization, measurement, and application in the catalog and Internet shopping environment. Journal of Retailing, Amsterdan, v. 77, n. 1, p. 39-56, 2001.

MAXHAM, J. G.; NETEMEYER, R. G. A longitudinal study of complaining customers' evaluations of multiple service failures and recovery efforts. Journal of Marketing, Chicago, v. 66, n. 4 , p. $57-71,2002$.

MAZURSKY, D.; JACOBY, J. Exploring the development of store image. Journal of Retailing, Amsterdan, v. 62, n. 2, p. 145-165, 1986.

MITTAL, V.; ROSS, W. T.; BALDASARE, P. M. The asymmetric impact of negative and positive attribute-level performance on overall satisfaction and repurchase intentions. Journal of Marketing, Chicago, v. 62, n. 1, p. 33-47, 1998. 
João Marques Teixeira \& José Mauro da Costa Hernandez

MONROE, K.B. Pricing: Making profitable decisions. New York: McGraw-Hill, 1979.

OLIVER, R. L. Cognitive, affective, and attribute bases of the satisfaction response. Journal of Consumer Research, Chicago, v. 20, n. 3, p. 418-430, 1993.

OLIVER, R. L. Satisfaction: a behavioral perspective on the consumer. New York: McGrawHill, 1997.

OLIVER, R. L. Whence consumer loyalty? Journal of Marketing, Chicago, v. 63, n. 4, p. 3344, 1999.

OLIVER, R. L.; SWAN, J. E. Consumer perceptions of interpersonal equity and satisfaction in transaction: a field survey approach. Journal of Marketing, Chicago, v. 53, n. 2, p. 21-35, 1989.

OLIVER, R. L.; RUST, R.T.; VARKI, S. Customer delight: foundations, findings, and managerial insight. Journal of Retailing, Amsterdan, v. 73, n. 3, p. 311-336, 1997.

RAVALD, A.; GRÖNROOS, C. The value concept and relationship marketing. European Journal of Marketing, Bingley, v. 30, n. 2, p. 19-30, 1996.

ROOK, D. W. The buying impulse. Journal of Consumer Research, Chicago, v. 14, n. 2, p. 189-199, 1987.

SÁNCHEZ-FERNÁNDEZ, R.; INIESTA-BONILLO, M.A. The concept of perceived value: A systematic review of the research. Marketing Theory, Thousand Oaks, v. 7, n. 4, p. 427451, 2007.

SHERRY, J. F. A socio-cultural analysis of a Midwestern flea market. Journal of Consumer Research, Chicago, v. 17, n. 1, p. 13-30, 1990.

SHETH, J.N.; NEWMAN, B.I.; GROSS, B.L. Why we buy what we buy: A theory of consumption values. Journal of Business Research, Amsterdan, v. 22, n. 2, p. 159-170, 1991. 
Valores de compra hedônico e utilitário: os antecedentes e as relações com os resultados do varejo

SLATER, S.F. Developing a customer value-based theory of the firm. Journal of the Academy of Marketing Science, New York, v. 25, n.2, p. 162-167, 1997.

SWAN, J. E.; OLIVER, R. L. Post purchase communications by consumers. Journal of Retailing, Amsterdan, v. 65, n. 4, p. 516-533, 1989.

SWEENEY, J.C.; SOUTAR, G.N. Consumer perceived value: The development of a multiple item scale. Journal of Retailing, Amsterdan, v. 77, n. 2, p. 203-220, 2001.

THALER, R. Mental accounting and consumer choice. Marketing Science, Hanover, v. 4, n. 3, p. 199-214, 1985.

TVERSKY, A.; SATTATH, S.; SLOVIC, P. Contingent weighting in judgment and choice. Psychology Review, Washington, DC, v. 95, n. 3, p. 371-384, 1988.

VASKE, J. J.; FEDLER, A. J.; GRAFE, A.R. Multiple determinants of satisfaction from a specific waterfowl hunting trip. Leisure Science, New York, v. 8, n. 2, p. 149-166, 1986.

WAKEFIELD, K. L.; BARNES, J. H. Retailing hedonic consumption: a model of sales promotion of a leisure service. Journal of Retailing, Amsterdan, v. 72, n. 4, p. 409-427, 1996.

WAKEFIELD, K. L.; BAKER, J. Excitement at the mall: determinants and effects on shopping response. Journal of Retailing, Amsterdan, v. 74, n. 4, p. 515-539, 1998.

WESTBROOK, R. A. Product/consumption-based affective responses and post purchase processes. Journal of Marketing Research, Chicago, v. 24, n. 3, p. 258-270, 1987.

WOODAL, T. Conceptualising 'value for the customer': An attributional, structural and dispositional analysis. Academy of Marketing Science Review, New York, v. 12, p. 1-42, 2003. 
João Marques Teixeira \& José Mauro da Costa Hernandez

WOODRUFF, R.B. Customer value: The next source for competitive advantage. Journal of the Academy of Marketing Science, New York, v. 25, n. 2, p. 139-153, 1997.

ZEITHAML, V. A. Consumer perceptions of price, quality, and value: a means-end model and synthesis of evidence. Journal of Marketing, Chicago, v. 52, n. 3, p. 2-22, 1988. 
Valores de compra hedônico e utilitário: os antecedentes e as relações com os resultados do varejo

\section{Anexo 1: Indicadores dos constructos da pesquisa}

\begin{tabular}{|c|c|c|}
\hline Constructo & Código & Indicadores \\
\hline Ambiente da Loja & NPAR & Cores das paredes e pisos \\
\hline \multirow[t]{4}{*}{ - Ambiente Físico } & NDEC & Decoração \\
\hline & NILU & Iluminação \\
\hline & NTEM* & Temperatura \\
\hline & NLIM* & Limpeza \\
\hline \multirow{4}{*}{$\begin{array}{l}\text { Ambiente da Loja } \\
\text { - Organização }\end{array}$} & NARR & Arrumação das mercadorias nas prateleiras \\
\hline & NLOC & Facilidade de localizar os produtos \\
\hline & NIDE* & Facilidade de identificar os preços dos produtos \\
\hline & NESP & Espaço para andar \\
\hline \multirow{2}{*}{$\begin{array}{l}\text { Ambiente da Loja } \\
\text { - Sortimento }\end{array}$} & NVMA & Variedade de marcas disponíveis \\
\hline & NVPR & Variedade de produtos disponíveis \\
\hline \multirow{3}{*}{$\begin{array}{l}\text { Ambiente da Loja } \\
\text { - Atendimento } \\
\text { dos Vendedores }\end{array}$} & NATE & Atendimento dos vendedores \\
\hline & NEVE & Tempo de espera para ser atendido pelos vendedores \\
\hline & NEPA & Tempo de espera para fazer o pagamento \\
\hline \multirow{7}{*}{$\begin{array}{l}\text { Valor de Compras } \\
\text { Hedônico }\end{array}$} & VH1 & Eu me diverti fazendo esta compra. \\
\hline & $\mathrm{VH} 2 *$ & Eu fiz esta compra porque quiz e não por obrigação \\
\hline & VH3 & $\begin{array}{l}\text { Comparando com outras coisas que eu poderia ter feito, o tempo gasto } \\
\text { comprando este produto foi muito mais agradável. }\end{array}$ \\
\hline & VH4 & Esqueci os meus problemas enquanto estive nesta loja. \\
\hline & VH5 & Fazer esta compra foi uma verdadeira distração para mim. \\
\hline & VH6 & Estar nesta loja foi um jeito muito agradável de passar o tempo. \\
\hline & $\mathrm{VH} 7 *$ & A busca por este produto me deu um grande prazer. \\
\hline \multirow{6}{*}{$\begin{array}{l}\text { Valor de Compras } \\
\text { Utilitário }\end{array}$} & VU1* & Eu fui eficiente ao fazer esta compra. \\
\hline & VU2 & Eu gastei apenas o tempo necessário para fazer esta compra. \\
\hline & VU3 & Eu fiz esta compra com rapidez. \\
\hline & VU4* & Fazer esta compra foi como cumprir uma obrigação. \\
\hline & VU5* & $\begin{array}{l}\text { Foi fácil fazer esta compra porque eu sabia exatamente o que eu queria } \\
\text { comprar. }\end{array}$ \\
\hline & VU6 & O preço que paguei nesta compra compensou o tempo que gastei. \\
\hline \multirow[t]{4}{*}{ Satisfação } & SAT1 & Comprar este produto foi uma das melhores coisas que eu poderia ter feito. \\
\hline & SAT2 & O produto que eu comprei era exatamente o que eu estava precisando. \\
\hline & SAT3 & Eu estou satisfeito com a minha decisão de ter comprado este produto. \\
\hline & SAT4 & Eu me sinto feliz por ter comprado este produto. \\
\hline \multirow[t]{3}{*}{ Boca a boca } & BB1 & Eu pretendo recomendar esta loja para os meus amigos e parentes. \\
\hline & BB2 & Eu pretendo falar bem sobre esta loja para as outras pessoas. \\
\hline & BB3 & Se alguém pedir meu conselho, eu pretendo recomendar esta loja. \\
\hline Intenção de & IR1 & Eu pretendo voltar a esta loja para fazer compras no futuro. \\
\hline \multirow[t]{2}{*}{ Recompra } & IR2 & Eu pretendo voltar a esta loja com mais frequiência. \\
\hline & IR3 & Eu pretendo aumentar o valor das minhas compras nesta loja. \\
\hline
\end{tabular}

*Indica items eliminados durante a análise 